
allemande

46-1 | 2014

Les fondements normatifs de l'État constitutionnel moderne en Allemagne. Une approche pluridisciplinaire

\title{
État et éducation religieuse
}

Une discussion sous la République de Weimar

\section{Antje Roggenkamp}

Traducteur : Philippe Büttgen

\section{(2) OpenEdition}

\section{Journals}

Édition électronique

URL : https://journals.openedition.org/allemagne/1220

DOI : $10.4000 /$ allemagne. 1220

ISSN : 2605-7913

\section{Éditeur}

Société d'études allemandes

Édition imprimée

Date de publication : 30 juin 2014

Pagination : 21-34

ISSN : 0035-0974

Référence électronique

Antje Roggenkamp, «État et éducation religieuse », Revue d'Allemagne et des pays de langue allemande [En ligne], 46-1 | 2014, mis en ligne le 29 juillet 2019, consulté le 19 mai 2021. URL : http:// journals.openedition.org/allemagne/1220; DOI : https://doi.org/10.4000/allemagne.1220 


\section{État et éducation religieuse Une discussion sous la République de Weimar}

\section{- Antje Roggenkamp*}

Dans une lettre de Schleiermacher à son successeur berlinois, August Twesten, on peut lire cette affirmation:

«La question principale que vous me posez, celle de l'enseignement religieux à destination des enfants, me semble être l'une des plus difficiles; je ne sais rien en dire, sinon négativement ${ }^{(1)}$.

Schleiermacher, on le sait, ne fut pas seulement l'un des plus grands philosophes, théologiens et pédagogues du $\mathrm{XIX}^{\mathrm{e}}$ siècle allemand; son influence s'étendit jusqu'à la conception des réformes éducatives de Wilhelm von Humboldt ${ }^{(2)}$. Il ne lui eût donc pas été difficile de modifier le système scolaire au lendemain de la lourde défaite subie par l'État prussien face aux armées napoléoniennes. Et pourtant: la religion faillit bien disparaître du corps des disciplines enseignées dans les écoles allemandes. Il s'en fallut de peu - très exactement, d'une concession de Schleiermacher à son mentor, le roi de Prusse Frédéric Guillaume I ${ }^{\text {er }(3)}$.

Sur ce point, les hésitations de Schleiermacher ont tenu moins à la crainte de voir l'enseignement religieux opérer une captation des esprits qu'à celle de jeter, par cette mesure même, le discrédit sur l'Église: en organisant un enseignement public de la

* Professeur de théologie pratique, Theologische Fakultät, Université de Göttingen.

1 Lettre de Friedrich Schleiermacher à August Twesten, 22 février 1812, in: Friedrich SchleiermaCHer, Texte zur Pädagogik. Kommentiere Studienausgabe, t. I, éd. Michael Winkler et Jens Brachmann, Francfort-sur-le-Main, Suhrkamp, 2000, p. 172.

2 Voir Dietrich Benner, Wilhelm von Humboldts Bildungstheorie, Weinheim, Juventa, 2003 (3e éd.).

3 Voir Christiane Ehrhardt, Religion, Bildung und Erziehung bei Schleiermacher. Eine Analyse der Beziehungen und des Widerstreits zwischen den 'Reden über die Religion' und den 'Monologen', Göttingen, Vandenhoeck \& Ruprecht, 2005, p. 230-231. 
religion, l'État revendiquait pour lui les compétences de l’Église, sans compenser cette prise en main organisationnelle par un surcroît de liberté ${ }^{(4)}$.

On peut donc comprendre que Schleiermacher se soit montré si ouvertement hostile aux «noces » de l'État et de l'Église ${ }^{(5)}$. Ce qui est en revanche plus difficile à expliquer, c'est ceci: après Schleiermacher, il fallut attendre encore plus de cent ans la séparation officielle de l'Église et de l'État. Ce n'est qu'en 1919 que la Constitution de Weimar abolit l'Église d'État ${ }^{(6)}$.

Et pourtant, à nouveau: jamais l'Allemagne n'est arrivée à une complète décléricalisation de son système scolaire public. En 1932, à la fin de la République de Weimar, non seulement les écoles confessionnelles s'étaient développées en nombre et en importance, mais l'enseignement de la religion s'était fermement établi comme discipline officielle dans la plupart des régions, à l'exception de Brême et de Hambourg ${ }^{(7)}$.

Les remarques qui suivent visent à définir les raisons qui ont rendu possible cette singulière évolution. La première partie s'intéressera moins au rôle joué par le «père » de la Constitution de Weimar, Hugo Preuß, qu'à la discussion intense provoquée par l'adoption de la première constitution démocratique en territoire allemand. Dans une deuxième partie, on s'intéressera aux diverses tentatives juridiques et philosophiques engagées alors pour redéfinir la relation entre Église et État. Enfin la troisième partie reconstituera la réception des dispositions constitutionnelles relatives à l'État et aux Églises dans plusieurs revues pédagogiques protestantes ${ }^{(8)}$. Les discussions à ce sujet n'allèrent pas sans comparaisons internationales, en particulier au début de la République de Weimar ${ }^{(9)}$. On insistera néanmoins surtout sur l'actualité que les modèles élaborés à cette époque ont conservée jusqu'à aujourd'hui, par-delà leurs évolutions.

\section{Les soubassements politiques}

La proclamation par Philipp Scheidemann (1865-1939), le 9 novembre 1918, d'une République parlementaire n'entraîne pas seulement la destruction del'Empire allemand (Kaiserreich) et de son régime politique ${ }^{(10)}$. En même temps que la monarchie constitutionnelle, ce sont toutes les structures administratives antérieures qui paraissent, dans

4 Cf. Friedrich Schleiermacher, Über die Religion. Reden an die Gebildeten unter ihren Verächtern, Kritische Gesamtausgabe, t. 1/2: Schriften 1796-1799, Berlin/New York, De Gruyter, p. 185-326, sp. 282-283.

5 Cf. p. ex. le Vorschlag zu einer neuen Verfassung der protestantischen Kirche für den preußischen Staat vom 18. November 1808 .

6 «Il n’y a pas d'Église d'État » : voir Verfassung des Deutschen Reiches. Vom 11. August 1919, \$137.1, in: Reichsgesetzblatt, Jg. 1919, Nr. 152, Berlin, 1919, p. 1383-1418, sp. 1409.

7 Voir dernièrement Rainer LachmanN, "Die Weimarer Republik », in: ID./Bernd Schröder (dir.), Geschichte des evangelischen Religionsunterrichts in Deutschland, Neukirchen, Neukirchener Verlag, 2007, p. 203-232, sp. 208-209.

8 Pour le catholicisme allemand, cf. Hans Mend,, "Katholischer Religionsunterricht - ein Längsschnitt ", in: LACHMAnN/SCHRÖDER (dir.), Geschichte des evangelischen Religionsunterrichts (note 7), p. 331-364, sp. 348.

9 Voir p. ex. Kurt Kesseler, Brauchen wir Religionsunterricht und welchen? Eine Apologetik und Methodik des evangelischen Religionsunterrichts, Leipzig, Klinkhardt, 1919, p. 8-9.

10 Cf. Kristian Klaus Kronhagel, Religionsunterricht und Reformpädagogik. Otto Eberhards Beitrag zur Religionspädagogik in der Weimarer Republik, Münster, Lit, 2004, p. 34. 
un premier temps, englouties. Le ministère prussien chargé de l'Instruction et des Cultes (Ministerium für geistliche und Unterrichtsangelegenheiten) s'appelle désormais ministère de la Science, de l'Art et de l'Éducation nationale (Ministerium für Wissenschaft, Kunst und Volksbildung). Pour des raisons différentes, les deux premiers titulaires du poste, Adolf Hoffmann (1858-1930), socialiste de gauche et athée déclaré, et le socialiste modéré Konrad Haenisch (1876-1925) s'attachent à affaiblir durablement l'enseignement religieux en Prusse. Hoffmann surtout œuvre dans le but déclaré d'empêcher toute modification du nouveau statu quo législatif fixé par les décrets des 15, 20 et 29 novembre 1918, qui dans une très large mesure excluent l'éducation religieuse de l'école publique ${ }^{(11)}$. Et de fait: pendant un bref moment, l'enseignement de la religion en Allemagne s'est retrouvé dans un vide juridique ${ }^{(12)}$.

Assez vite toutefois, ce traitement (politique) de l'éducation religieuse semble avoir indisposé de larges pans de la population, au point de peser de manière non négligeable sur la préparation des échéances électorales ${ }^{(13)}$. Les élections des 19-26 janvier 1919 modifient significativement le rapport de forces politique à l'échelle du territoire entier. En lieu et place d'une « République des conseils » s'installe la " coalition de Weimar» formée par le SPD, le DDP libéral et le Zentrum catholique. En matière scolaire, les partis de la coalition parviennent à deux compromis ${ }^{(14)}$. Le premier, conclu le 19 juin 1919, prévoit la possibilité de fonder à la fois des écoles « laïques » et des écoles confessionnelles (\$146-2 de la Constitution de Weimar). Les revendications respectives du SPD (école neutre), du DDP (école non-confessionnelle) et du Zentrum (école confessionnelle) se trouvaient ainsi satisfaites ${ }^{(15)}$. Le second compromis des 30-31 juillet 1919 porte en particulier sur l'enseignement de la religion. La mention des deux disciplines de l'éducation civique et de la "formation au travail " (Arbeitsunterricht) ${ }^{(16)}$ dans la nouvelle Constitution $(\$ 148-3)$ permet le maintien de la religion dans son rang de discipline officielle de toutes les écoles publiques $(\$ 149-2)^{(17)}$.

11 Ibid., p. 41.

12 Ibid., p. 215.

13 Sur tout cela, on se référera toujours à Peter C. Bцотн, Religion in den Schulen Preußens, Heidelberg, Quelle und Meyer, 1969, p. 180-181. Voir aussi K. KRONHAGEL, Religionsunterricht und Reformpädagogik (note 10), p. 30-46.

14 Voir sur ce point Axel Frhr. von Campenhausen, « Der heutige Verfassungsstaat und die Religion ", $\$ 2$, in: Dietmar Pirson, Joseph Listu (éd.), Handbuch des Deutschen Staatskirchenrechts, t. 1, Berlin, Duncker \& Humblot, 1994 (2 éd.) p. 47-84, sp. 63-64, ainsi que Kurt NowaK, Evangelische Kirche und Weimarer Republik. Zum politischen Weg des deutschen Protestantismus zwischen 1918 und 1932, Göttingen, Vandenhoeck \& Ruprecht, 1988 (2 éd.), p. 81-82. Autre point de vue dans K. KronHAGEL, Religionsunterricht und Reformpädagogik (note 10), p. 61-65.

15 Cf. P.C. Bцотн, Religion in den Schulen Preußens (note 13), p. 188, ainsi que K. NowaK, Evangelische Kirche und Weimarer Republik (note 14), p. 81.

16 Au début de la République de Weimar, cette nouvelle discipline avait pour but d'orienter les élèves vers une formation éthique. Vers 1926, la " formation au travail » est considérée plutôt comme un principe didactique et méthodique; dans les Richtlinien für die Deutsche Oberschule (1924), document important pour l'enseignement secondaire en Prusse, elle relie diverses disciplines : allemand, histoire, éducation religieuse. Voir Antje Roggenkamp, "Reformpädagogik in religionspädagogischen Zeitschriften protestantischer Provenienz », in: Michael Wermкe (dir.), Religionspädagogik und Reformpädagogik. Brüche, Kontinuitäten, Neuanfänge, Iéna, Édition Paideia, 2010, p. 113-138, sp. p. 126 sqq.

17 Sur la diversité de situations sur le territoire du Reich, cf. Monatsblätter für den evangelischen Religionsunterricht (abr. MERU), Göttingen, Vandenhoeck \& Ruprecht, 12 (1919), p. 38-40. 
L'entrée en vigueur de la Constitution de Weimar le 11 août 1919 fait de la République un État séculier de iure ${ }^{(18)}$; le détail des dispositions relatives au rapport entre Église et école est renvoyé à une future loi scolaire du Reich $(\$ 174)^{(19)}$. Du côté protestant, c'est la réception des articles scolaires de la Constitution de Weimar par les partis proches de l'Église, DNVP et DVP, qui a fait l'objet du plus grand nombre d'études ${ }^{(20)}$. J'examinerai pour ma part la manière dont le rôle de l'État est théorisé dans le discours de l'époque. De ce fait, je m'intéresserai moins à l'expression officielle de l'Église qu'à la réalité plus vaste des diverses propositions juridiques et philosophiques relatives à l'enseignement religieux.

\section{Implications juridiques et philosophiques}

Dans les années qui précèdent et qui suivent l'entrée en vigueur de la Constitution de Weimar, l'éducation religieuse s'affronte principalement à deux problèmes. Il lui faut d'abord, pour ainsi dire en amont, reposer le problème ecclésiologique du rapport entre l'Église et l'État: de fait, si l'Église d'État a disparu, il existe désormais une garantie étatique accordée aux écoles liées aux Églises ainsi qu'à l'enseignement confessionnel. En aval s'impose la nécessité de repenser juridiquement et philosophiquement la situation et les tâches de l'éducation, y compris de l'éducation religieuse.

Peu avant la naissance de la première République démocratique sur le sol allemand, plusieurs modèles théoriques ont été tracés pour formaliser les relations entre l'Église et l'État ${ }^{(21)}$. Ces modèles ont trouvé leur approfondissement dans la réflexion sur le droit des Églises. La préoccupation principale ne tient pas tant ici aux conséquences concrètes de la séparation de l'Église et de l'État qu'à la volonté de désactiver intellectuellement cette séparation avant même qu'elle n'entre en vigueur. Les premiers écrits de Friedrich Gogarten (1887-1967) renvoient ainsi l'État vers l'Église pour lui permettre, contre les tentations d'un libertinisme individualiste ou d'un rationalisme de la personne, de déployer sa pleine souveraineté(22). Chez le juriste Günter Holstein (1892-1931), membre de l'Église de l'Union, l'accent de la réflexion juridico-ecclésiologique est mis à l'inverse sur l'Église ${ }^{(23)}$. Certes Holstein oppose «l'Église de l'Esprit » et « l'Église du droit ». Entre elles cependant, il ouvre l'espace d'une Église conçue comme « communauté sociale », qui permet de penser l'État comme garant positif de la liberté de l'Église ${ }^{(24)}$. Une argumentation similaire se retrouve chez le dogmaticien Otto Piper (1891-1982) avant son départ pour les États-Unis : son refus d’une politisa-

18 Voir notamment K. NowaK, Evangelische Kirche und Weimarer Republik (note 14), p. 72 sqq.

19 Sur les différents projets de loi scolaire, cf. P.C. Bцотн, Religion in den Schulen Preußens (note 13).

20 Voir notamment P.C. Bloth, Religion in den Schulen Preußens (note 13), p. 188 sqq.

21 Très instrutif sur les problématiques juridiques liées au statut des Églises dans l'État, Eckard LEssiNG, Geschichte der deutschsprachigen evangelischen Theologie von Albrecht Ritschl bis zur Gegenwart, t. 1: 1870-1918, Göttingen, Vandenhoeck \& Ruprecht, 2000, p. 462-484.

22 Voir p. ex. Friedrich Gogarten, Religion und Volkstum, Iéna, 1915 avec en arrière-fond l'hostilité envers la Révolution française. Cf. K. Nowak, Evangelische Kirche und Weimarer Republik (note 14), p. 238-239.

23 Sur Holstein, voir K. NowaK, Evangelische Kirche und Weimarer Republik (note 14), p. 218-219.

24 Cf. Eckard Lessing, Geschichte der deutschsprachigen evangelischen Theologie von Albrecht Ritschl bis zur Gegenwart, t. 2: 1918-1945, Göttingen, Vandenhoeck \& Ruprecht, 2004, p. 408-409. 
tion de l'Église n'a d'égale que sa volonté de promouvoir son ouverture au monde ${ }^{(25)}$. L'opposition que Piper établit dans ce domaine entre les formes d'une argumentation empirique et d'une argumentation transcendantale repose sur la conviction qui est la sienne que seule la créature comme créature peut revendiquer un accès au réel. En d'autres termes, seule la croyance en la création peut faire de l'Église une réalité concevable ${ }^{(26)}$.

La relation Église-État a ainsi fait l'objet d'une discussion intense dans le droit des Églises d'avant 1919. Il n'en va pas de même dans le droit public. Ce n'est qu'au début du siècle qu'on met au point des outils théoriques à ce sujet, en faisant appel pour l'essentiel à d'anciens modèles de philosophie politique ${ }^{(27)}$. Chez Kant, l'analyse historique des interactions concrètes entre Église et l'État repose non pas sur la considération de l'entité abstraite de l'État, mais bien plutôt sur la détermination sociologique de ces interactions. À la différence de ce qu'on observe chez les théoriciens du contrat qui l'ont précédé, ce n'est pas l'homme, mais le citoyen que Kant place au centre de sa réflexion sur le sujet. Sa fondation rationnelle de la politique fait de l'État un système de législation publique. L'État, par conséquent, est défini par Kant comme la communauté des hommes rassemblés sous les lois juridiques. Sa première mission est de garantir juridiquement la liberté et l'égalité de ses citoyens, en s'appuyant sur les droits fondamentaux de tout homme, tels qu'ils sont garantis par les postulats en dernière instance religieux de la Critique de la raison pratique ${ }^{(28)}$.

La réflexion de Hegel sur les rapports de l'Église et de l'État s'ancre aussi dans un concept de liberté. Contre Kant toutefois, Hegel conçoit le droit comme l'expression d'une volonté de liberté. L'État est donc la réalisation de l'Idée éthique, que Hegel ramène à des normes juridiques supra-positives puisées dans le christianisme. Si la religion ne peut comme telle prétendre régir l'État, celui-ci en revanche requiert, au moment même où il s'agit de rendre compte de sa sécularité, la claire conscience de ses liens avec la religion et l'esprit de la religion ${ }^{(29)}$. La philosophie politique de Hegel a marqué de son empreinte toutes les conceptions de l'État comme organisme suprahumain, toutes ses personnifications aussi ${ }^{(30)}$.

25 Sur Piper, voir K. NowaK, Evangelische Kirche und Weimarer Republik (note 14), p. 214-215.

26 Otto Piper, Theologie und reine Lehre, Tübingen, 1926, p. 199: " L'Église est l'efficace de Dieu sur terre".

27 Cf. Michael Stolleis, «Staatsethik», in: Evangelisches Kirchenlexikon, t. 4, Göttingen, Vandenhoeck \& Ruprecht, 1996, p. 461-469, sp. 465.

28 Cf. Wolfgang Kersting, art. «Staat/Staatsphilosophie», in: Theologische Realenzyklopädie, t. 32, Berlin/New York, De Gruyter, 2001, p. 51 sq. Voir aussi Friedrich KaulBaCH, Immanuel Kant, New York/ Berlin, De Gruyter, 1982 (2 éd.), p. 239-243, p. 291 sqq.

29 Hegel, Grundlinien der Philosophie des Rechts, $\$ 270$, Remarque, dans l'éd. de Jean-François KervéGAN, Principes de la philosophie du droit, Paris, PUF (Quadrige), 2003 (2 éd.), p. 352-363.

30 Cf. W. Kersting, «Staat/Staatsphilosophie» (note 28), p. 53-54. Les deux positions de Kant et Hegel se reflètent dans ce qu'on a appelé la « querelle du droit public » à Weimar, et notamment dans l'opposition entre le néo-kantien Hans Kelsen (1881-1973) et le néo-hégélien Hermann Heller (1891-1933): voir ibid., p. 55-56. 


\section{Les discours de la pédagogie religieuse}

On envisagera à présent la question du rôle de l'État dans l'éducation religieuse au travers des prises de position de divers représentants officiels de la discipline appelée «pédagogie religieuse " (Religionspädagogik) ${ }^{(31)}$. Les textes que je mentionnerai sont extraits des deux grandes revues de pédagogie religieuse protestante; ils reflètent à ce titre l'orientation majoritaire du corps des professeurs de la discipline ${ }^{(32)}$.

\section{Avant 1918: les fondations}

La réflexion sur la séparation de l'Église et de l'État n'a pas commencé en novembre 1918. Dès 1908, le duisbourgeois Friedrich Feigel (né en 1875), qui allait devenir le président de l'Association rhénane des professeurs de religion, évoque « L'enseignement de la religion à l'école en lutte pour sa survie » ${ }^{(33)}$. Feigel plaide pour une approche historique de l'enseignement de la religion, rendue selon lui nécessaire par l'illégitimité qui frappe depuis Kant toute forme dogmatique d'éducation religieuse: "traiter les postulats de la raison pratique comme des résultats théoriques, c'est faire peu de cas de la vérité » ${ }^{(34)}$. Dans ces conditions, l'État a un double intérêt à l'existence d'un enseignement de la religion. Celui-ci, dès lors qu'il fait l'objet d'un traitement réflexif, entre d'abord de plein droit dans les tâches éducatives de l'école ${ }^{(35)}$. En second lieu, cet enseignement doit prendre en charge l'éducation éthique de la population: "c'est l'intérêt de l'État qu'existe un enseignement religieux à l'école, à condition qu'il n'hésite pas à lui confier largement la réalisation des tâches éthiques que l'État moderne a faites siennes » ${ }^{(36)}$.

En 1912, Heinrich Spanuth (1873-1958), éditeur des Monatsblätter für den evangelischen Religionsunterricht, directeur du Séminaire de Hameln et représentant de l'Alliance pour l'éducation et l'enseignement religieux (Bund für religiöse Erziehung und Religionsunterricht, BRRU), plaide en toute rigueur de termes pour " la décléricalisation de l'État et la désétatisation de l'Église " ${ }^{(37)}$. Il renonce à fonder l'intérêt étatique pour un enseignement de la religion sur "l'une des théories issues de la philosophie politique ». L'État comme organisateur du système éducatif n'a qu'un « intérêt extrinsèque [...] à la diffusion d'un contenu déterminé d'enseignement

31 La question des rapports avec l'État n'est pas traitée pour elle-même dans les revues pédagogiques catholiques; elle constitue bien la signature de la réflexion protestante sur l'éducation. Cf. Henrik Simojoki, Friedrich Schweitzer, Moderne Religionspädagogik. Ihre Entwicklung und Identität, Gütersloh, Gütersloher Verlagshaus, 2005, p. 178-182, 185.

32 J'ai consulté principalement la Zeitschrift für den evangelischen Religionsunterricht, fondée en 1890, et les Monatsblätter für evangelischen Religionsunterricht (1909), en laissant de côté d'autres revues du domaine comme la Evangelische Pädagogik, dont la fondation intervient après 1925. Voir Gury Schneider-Ludorff, Magdalene von Tiling. Ordnungstheologie und Geschlechterbeziehungen - Ein Beitrag zum Gesellschaftsverständnis des Protestantismus in der Weimarer Republik, Göttingen, Vandenhoeck \& Ruprecht, 2001. MERU, 1 (1908), p. 137-142; p. 175-185. Ibid., p. 141-142. Ibid., p. 175: « l'objectif principal que l'école doit remplir pour le peuple et l'État, c'est l'instruction ». Ibid., p. 180.

Heinrich Spanuth, "Staat und Kirche im Religionsunterricht der Schule. Vortrag gehalten auf der Religionslehrerkonferenz des Oldenburgischen Landeslehrervereins am 23. Dezember 1911 ", $M E R U$, 5 (1912), p. 33-43. 
religieux " ${ }^{(38)}$. Pour cette raison, la revendication, non seulement d'un modèle scolaire unique à l'échelle de la nation, mais même de toute forme d'école unique, quelle qu'elle soit, doit être résolument rejetée ${ }^{(39)}$. L'État doit veiller à ce que la diversité de ses citoyens se retrouve à l'intérieur de l'école: " plus grande sera la diversité, et plus il y aura de force et de vie, plus riche sera le produit de toutes les forces créatrices de vie dans l'État " ${ }^{(40)}$. Spanuth, contrairement à Feigel, récuse donc aussi la revendication d'un enseignement purement informatif et historique de la religion, de la même façon qu'il écarte l'exigence d'un enseignement purement éthique. Si Spanuth a d'emblée renoncé au choix d'une philosophie politique particulière, la manière dont il justifie l'existence d'un enseignement confessionnel donne une idée de son orientation:

" "Être neutre” pour l'État, cela ne signifie pas être incolore ou promouvoir l'incolore dans l'enseignement. Cela signifie que l'État procure à tous les citoyens le droit et la liberté dont ils ont le besoin et le désir. De ce point de vue, il n'y a aucune contradiction à revendiquer la protection par l'État d'un enseignement religieux "confessionnel" » ${ }^{(41)}$.

En 1916, l'expérience de la guerre va amener Spanuth à opérer une légère inflexion. Si l'intensification du sentiment national l'incite, comme plus tard Schuster, à repenser les rapports entre religion et patriotisme, il recommande aussi de prendre en compte le « caractère supranational » du christianisme, autrement dit d'assumer l'« élément étranger » des autres confessions et de le mettre en relation avec le " propre » de chacun, de façon à ce que ces deux éléments, loin de se combattre, entrent en complémentarité $^{(42)}$. De cette interprétation révisée de la réalité historique pourraient, selon lui, bénéficier aussi bien les croyants d'autres confessions que les citoyens sans religion ${ }^{(43)}$.

C'est sans doute Hermann Tögel (1869-1939), professeur à Leipzig et futur membre du directoire du BRRU, qui adopte le propos le plus général en s'affrontant aux thèses (en apparence) positivistes du célèbre pédagogue berlinois, le néo-kantien Friedrich Paulsen ${ }^{(44)}$. Ignorant les conséquences pédagogiques des «sécularisation et étatisation progressives du système éducatif », que Paulsen plaçait en parallèle de la démocratisation ou plutôt de l'inévitable ouverture de l'enseignement ${ }^{(45)}$, Tögel formule l'exigence d'un refus par l'État de «toute forme d'enseignement religieux qui se situerait entre les Églises, ou au-delà des Églises ». La nouvelle direction prise par l'âme allemande en matière religieuse se détourne selon lui d'un passé formaté par la rivalité confessionnelle. Les espoirs de Tögel vont vers un enseignement protestant librement offert à tous, un enseignement qui, «par la vertu des valeurs allemandes éternelles qui reposent en

38 Ibid., p. 38.

39 Ibid., p. 39.

40 Ibid.

41 Ibid., p. 43.

42 Heinrich SPANUTH, « Wie wirkt der Krieg bereichernd und umgestaltend auf den Religionsunterricht ein?», MERU, 9 (1916), p. 154-157, sp. 156.

43 ID., « Der Einfluß des Weltkrieges auf den Kampf um den Religionsunterricht der Schule », MERU, 9 (1916), p. 266-269, sp. 269.

44 Hermann Tögel, "Die Entwicklung des evangelischen R.-U. nach dem Weltkriege », MERU, 10 (1918), p. 14-19.

45 Friedrich PAulsen, Das deutsche Bildungswesen in seiner geschichtlichen Entwicklung, Leipzig, 1912, p. 171. 
lui » ${ }^{(46)}$, attirerait à lui toutes les confessions, de façon à sceller l'alliance définitive du christianisme de la Réforme et de l'âme nationale allemande ${ }^{(47)}$.

Dès 1917, l'éditeur de la Zeitschrift für den evangelischen Religionsunterricht, Hermann Schuster (1874-1965), président de l'Association allemande des professeurs de religion et futur député (DVP) au Parlement de Prusse, envisage l'émergence, à partir de l'expérience de la guerre, d'une nouvelle éthique reliant les problématiques chrétiennes et nationales. Ces réflexions prolongent l'accent mis en 1915 par Gogarten sur la question de la faute et son origine dans la liberté humaine: «l'animal ne connaît pas la faute; l'homme seul s'élève par la faute ${ }^{(48)}$. Schuster reprend la comparaison par Gogarten des deux images du monde séparées par l'événement de la Réforme: selon lui, la doctrine protestante des deux règnes a « fait tomber [...] l'ancienne séparation des deux gouvernements [sc. temporel et spirituel] et avec elle l'opposition absolue de Dieu et du monde ». En revanche, il critique chez Gogarten l'identification du christianisme avec l'" augustinisme, qui ramène tout au péché originel et au pessimisme ", alors qu'il faudrait selon lui considérer le second comme une simple variante du premier ${ }^{(49)}$. Cette identification rendrait impossible la définition d'un « regard contemporain sur le christianisme », un « regard moderne sur l'État et le peuple " ${ }^{(50)}$.

Ce qui frappe dans ces réflexions d'avant 1919 sur le rôle de l'État dans l'enseignement religieux, c'est l'absence totale de référence juridique; le droit des Églises y joue un rôle insignifiant. Pour l'essentiel, nos auteurs travaillent avec la philosophie politique de Kant. Leurs objectifs sont divers. Pour les uns, l'État doit se servir d'un enseignement historique de la religion pour affermir ses propres fondations. Pour les autres, la mise en place d'un enseignement confessionnel doit permettre d'intégrer la pluralité des confessions et des religions. Au bout du compte, l'idée d'un enseignement religieux relié à un avenir national-allemand et chrétien-protestant laisse peu de place à la liberté de l'individu ${ }^{(51)}$.

\section{Les «nouveaux départs" de la Constitution de Weimar}

En 1919, Kurt Kesseler (né en 1884), futur représentant de l’Association des professeurs de religion, inverse la formule d'Adolf Hoffmann selon laquelle la religion est " affaire privée »: selon lui, la religion est au contraire un facteur de développement de la culture et de la civilisation ${ }^{(52)}$. Si l'on peut admettre que l'appartenance à une com-

46 H. Tögel, «Die Entwicklung des evangelischen R.-U. » (note 44), p. 18.

47 Ibid., p. 19: "Le catholicisme et le protestantisme ne sont pas deux variantes équivalentes du christianisme. Le premier constitue une forme romaine de la religion chrétienne, celle par laquelle l'esprit national romain a dominé les peuples germaniques au Moyen Âge, celle par laquelle il continue à peser sur eux. C'est la religion du passé. La Réforme allemande constitue la prise en charge du christianisme par l'âme nationale allemande. C'est la religion de l'avenir. »

48 Hermann Schuster, «Umschau. Neue Aufgaben: Religion und deutsches Volkstum, Christentum und deutscher Staat ", Zeitschrift für den evangelischen Religionsunterricht (abr. ZevRU), Berlin, Reuther und Reichard, 28 (1917), p. 31-40, sp. 34.

49 Cf. Hans Georg Drescher, art. "Friedrich Gogarten », in: Die Religion in Geschichte und Gegenwart, t. 3, col. 1071-1072, New York/Berlin, De Gruyter, 2000 (4 éd.).

50 H. Schuster, «Umschau » (note 48), p. 35.

51 Voir sur ce point Simojoki/Schweitzer, Moderne Religionspädagogik (note 31), p. 79 sqq.

52 K. Kesseler, Brauchen wir Religionsunterricht und welchen? (note 9), p. 8-9. 
munauté déterminée constitue de fait une affaire privée, en revanche, dès lors que c'est précisément du point de vue éthique que la religion (chrétienne) constitue un «facteur civilisationnel décisif », l'État ne peut être indifférent à la question de savoir s'il existe ou non une ou des religions ${ }^{(53)}$.

Dès 1924, Kesseler esquisse une nouvelle ligne d'argumentation. La relation entre religion et culture doit être caractérisée d'une manière qui intègre les apports de la philosophie de la religion et de la philosophie de l'histoire, mais qui sache aussi se démarquer de ces approches ${ }^{(54)}$. Il faut saisir la religion comme une forme spécifique de rapport au monde: elle est l'âme de toute culture, mais elle est aussi son juge, qui convoque devant son tribunal tous les errements d'une culture livrée à l'illusion de son autonomie. Mais la religion ne peut s'acquitter de cette tâche que si son existence n'est pas seulement assurée par des arrangements empiriques et plus ou moins aléatoires, mais bien en elle-même et pour elle-même. Or toute religion fait, par essence, communauté, et sa valeur n'est véritablement comprise que lorsqu'on intègre sa dimension sociale. Pour cette raison, les questions d'éthique sociale et de pédagogie chrétienne doivent prendre le pas sur les questions dogmatiques ${ }^{(55)}$. Le problème est néanmoins que la question de la vérité, qui reste au centre de toute justification de la religion, ne peut être laissée de côté. Kesseler cherche une solution du côté d'une réappréciation générale du rapport entre religion et esprit (humain) au sein d'une philosophie religieuse rénovée. D’ici à ce que cette tâche soit complètement réalisée, et aussi bien dans l'optique d'une « validation de la théologie » que dans celle d'un " renforcement de la vie religieuse ", il faut selon lui faire en sorte que la religion soit soutenue dans l'exercice des fonctions spécifiques qu'elle remplit au service de la culture ${ }^{(56)}$.

À son tour le puissant président du BRRU Hans Schlemmer (né en 1885) s’interroge en 1924 sur la place du concept de communauté dans l'enseignement de la religion ${ }^{(57)}$. Pour lui la situation historique se caractérise d'abord par la poussée des aspirations populistes:

« Je n’ai pas besoin d'insister sur le fait que l'appel de la liberté a laissé la place, dans la vie politique, à un désir de dictature [...] Les mouvements de jeunesse "bourgeois", au départ radicalement hostiles à toute forme de contrainte politique, passent maintenant en rangs serrés dans le camp nationaliste ${ }^{(58)}$.

Lui-même pourtant montre peu d'enthousiasme à développer une interprétation théologique des notions de peuple ou de nation. Il doute même que le protestantisme puisse accéder au statut de religion nationale:

53 Ibid.

54 Se réclamant de plus en plus clairement de la philosophie de l'histoire de Hegel, Kesseler s'oppose à la fois à Theodor Lessing, dont il critique l'orientation positiviste, et à Spengler, qui selon lui rompt l'unité du continuum historique: cf. "Kulturproblematik und Religionsproblematik », MERU, 17 (1924), p. 238-252, sp. 238-239. Ibid., p. 245.

56 Ibid., p. 252. Voir aussi ID., Religionsphilosophie, Düsseldorf, 1927.

57 Hans Schlemmer, "Freiheit des Religionsunterrichts und Freiheit im Religionsunterricht ", MERU, 17 (1924), p. 221-230, sp. 221. 
" Il y a une chose que je sais: on ne devient protestant qu'à la manière que je viens de décrire, celle qui vous arrache toutes vos béquilles et à force de trouver par vous-mêmes votre chemin vers Dieu. Tout le reste n'est que cryptocatholicisme, et nous devrions au moins éviter d'en faire notre idéal ${ }^{(59)}$.

Les temps présents requièrent selon Schlemmer de supporter autant que possible la tension qui se fait jour entre individualisme protestant et nationalisme cryptocatholique. La solution se laisse entrevoir dans l'espoir eschatologique « de nous voir tous réunis dans l'ecclesia possidentium comme dans l'ecclesia triumphans " ${ }^{(60)}$.

Le même malaise se retrouve en 1925 chez Friedrich Feigel. Nulle part « dans tout notre peuple ", dit Feigel, on n'entrevoit de dispositions et d'aspirations religieuses nouvelles. La nouvelle théologie conçue pour surmonter la crise spirituelle de l'époque lui paraît également sans issue: Karl Barth (1886-1968) et plus encore Friedrich Gogarten viennent alors d'affirmer l'existence d'une opposition absolue entre Dieu et la culture ${ }^{(61)}$. La forme de théologie négative qu'ils défendent rend superflu tout travail éducatif en matière religieuse. Le constat semble à Feigel d'autant plus vrai que, même si l'on fait abstraction de la surenchère théorique de Barth et Gogarten, la jeunesse paraît séparée du religieux par un abîme. Pour lui, la faute en revient à une interprétation particulière du christianisme:

«Une bonne partie de l'échec rencontré par le travail éducatif en matière religieuse vient de ce qu'on a essayé de tirer une règle, un schéma, de l'expérience classique des génies religieux et qu'on s'est attaché à mener des jeunes gens à la pénitence et à la foi par le dévoilement de leurs péchés, sur le modèle de Paul, de Luther et des piétistes » ${ }^{(62)}$.

Il importerait de parvenir à une compréhension plus réaliste de ce que sont les jeunes âmes: l'éducation religieuse devrait s'adresser aux jeunes "normaux ", ceux qui, à la différence des born again, ne sont « nés qu'une seule fois » (William James). Feigel préconise une approche progressive, qui fait de l'élément éthique une étape préparatoire à un enseignement de nature historique. L'application de cette démarche à l'enseignement religieux permettrait de construire progressivement une compréhension spécifique de la religion et de la culture ${ }^{(63)}$. Contre le « dénigrement » de cette dernière, les protestants seraient par conséquent bien inspirés d'en appeler à une culture mieux comprise, c'est-à-dire, au-delà, à une réflexion plus réaliste qui intégrerait le souci de l'action éthique:

«Dans ce combat, les cercles qui revendiquent une forte attache doctrinale dans l'enseignement religieux, finiront par faire front avec le catholicisme. Ce que je veux dire par là trouve un bon exemple dans le dernier succès remporté par le catholicisme dans sa guerre culturelle: au cours de l'année du bicentenaire de la naissance de Kant [1724, NdT], Richert a pu le plus ouvertement du monde faire de Kant le spiritus rector de nos universités, et avec lui l'idéalisme, "au centre duquel se trouve Kant" " ${ }^{(64)}$.

59 Ibid., p. 228.

60 Ibid.

61 Friedrich Feigel, "Theologische Gegenwartsnot des Religionsunterrichts », MERU, 18 (1925), p. $50-58$, sp. 51 .

62 Ibid., p. 53.

63 Ibid., p. 57-58.

64 Ibid., p. 58. 
Une telle insistance sur la liberté religieuse de l'individu ne peut tenir face aux attaques politiques à attendre de l'alliance cléricale avec l'État. Le raisonnement de Feigel s'achève sur cette note presque dramatique:

«Il serait temps qu'un nouveau Schleiermacher se lève pour lancer cette fois des "Discours sur la culture aux plus cultivés de ses détracteurs" ${ }^{(65)}$.

Le modèle originellement kantien d'un rapport entre religion et culture saisi dans les termes du droit individuel, garanti par l'État, à pratiquer librement sa religion et par conséquent à s'y former, ce modèle a été mis à mal par les tensions conceptuelles des années vingt entre religion et culture, peuple et individu. L'alliance des Églises revendiquant, dans leur rapprochement avec l'État, le retour à un ancrage confessionnel de l'enseignement religieux lui a été fatal. La question se pose de savoir quelle conception de l'État a pu servir aux théoriciens de l'enseignement protestant dans la légitimation de leur pratique professionnelle ${ }^{(66)}$.

\section{Après 1930: le protestantisme devant le monde}

C'est dans ce contexte que le philosophe et pédagogue Robert Scherwatzky (né en 1889), représentant du BRRU, attire l'attention de ses lecteurs de 1931 sur le phénomène dit de la " renaissance hégélienne ». La vie intellectuelle allemande a connu, selon Scherwatzky, un bouleversement "épouvantable " au cours de la Première Guerre mondiale. Les solutions du XIX ${ }^{\mathrm{e}}$ siècle, à commencer par celles de Kant, ont dû être rejetées ou ont révélé leur insuffisance. Il reste possible de s'orienter à partir de la question kantienne, "Que m’est-il permis d'espérer? ", mais celle-ci fait l'objet d'un traitement nouveau: Dieu est celui « vers qui l'homme peut se tourner dans sa misère terrestre ${ }^{(67)}$.

La renaissance hégélienne s'explique ici par les traits caractéristiques d'une pensée qui réunit l'attitude religieuse à l'ouverture au monde, ouvrant l'espace de la formation (ou auto-formation) religieuse nécessaire à l'individu ${ }^{(68)}$. Dans la question des relations entre la religion et l'État, Hegel, parce qu'il a pris une voie différente de celle de Schleiermacher ${ }^{(69)}$, apporte un argument important à la thèse d'une réciprocité,

65 Ibid.

66 Après 1924, la position kantienne fut certes de nouveau discutée, mais plus en ce qui concerne la question de l'éducation religieuse: cf. Kurt Kesseler, "Der Ertrag der Kantischen Religionsphilosophie », MERU, 21 (1928), p. 6-18, sp. 18, ainsi que Robert SCHERWATZKY, " Hegel in der religiösen Krisis der Gegenwart », MERU, 24 (1931), p. 265-268, sp. 266.

67 R. ScherWATZKY, « Hegel in der religiösen Krisis der Gegenwart » (note 66), p. 265-266: « Au plus profond de tous ces mouvements, on trouvera le renouveau religieux de la réflexion: le retour à l'Absolu constitue l'expression philosophique d'une nouvelle orientation vers le divin [...] Ces caractères ne sont pas tous récents, mais en eux réémergent d'anciennes idées que la coulée de boue du matérialisme avait englouties mais qu'il n'avait pas tuées. Ce n'est que maintenant que notre regard peut de nouveau s'ouvrir à Hegel, dont l'ouverture au monde (une éthique matériale des valeurs qui certes n'a rien à voir avec le matérialisme) et l'attitude religieuse sont particulièrement remarquables : ce n'est pas sans raison que nous parlons d'une renaissance hégélienne. "

68 Ibid., p. 268: "À la question: pourquoi suis-je là?, Hegel donne cette réponse: pour t’intégrer à la totalité harmonieuse de l'humanité [...]».

69 Voir plus haut, note 5, ainsi que K. Kesseler, "Kulturproblematik und Religionsproblematik " (note 54), p. 245. 
plutôt que d'une dépendance, entre État et enseignement religieux. La religion d'un peuple est étroitement liée à sa culture et à sa philosophie: "Dans le débat brûlant des rapports de la religion et de l'État, Hegel aborde la question de la séparation d'une façon très différente de celle de Schleiermacher: "La constitution d'un peuple est très étroitement liée à sa religion, à son art et sa philosophie" " ${ }^{(70)}$. Dans une période de crise qui, selon l'observation de pédagogues comme Spranger et Natorp, se déploie sur le terrain de la religion plus que de la politique, les conceptions politiques de Hegel se révèlent tout sauf obsolètes: « telle la religion, tels sont aussi l'État et la constitution; il n'y a pas de sens à construire des constitutions dans le vide, indépendamment des conditions spirituelles qu'elles puisent dans le peuple lui-même ${ }^{(71)}$.

Scherwatzky n'est pas le seul à faire sien le choix hégélien d'une "synthèse » de culture et de religion chrétienne à l'intérieur de l'enseignement religieux. Kesseler esquisse une argumentation similaire ${ }^{(72)}$. Contre eux, le pédagogue hessois Heinrich Matthes (1868-1931) fait valoir les inconvénients du passage de Kant à Hegel, c'est-àdire, en pédagogie, du subjectif à l'objectif ${ }^{(73)}$ : en lieu et place de l'« expérience personnelle », c'est la « Parole de Dieu » qui se retrouve au centre ${ }^{(74)}$.

Du point de vue institutionnel, la majorité des théoriciens de l'enseignement religieux adoptent à ce moment l'orientation hégélienne : l'État (plus ou moins personnifié) doit pour eux permettre la coexistence la moins conflictuelle possible de la religion et de la culture. Le luthérien Otto Eberhard (1875-1966), réformateur de la pédagogie et subtil interprète du premier compromis scolaire de Weimar, affirme ainsi que la tendance à l'étatisation, c'est-à-dire à la décléricalisation de l'école ne peut être arrêtée. La séparation juridique de l'Église et de l'État en est selon lui la conséquence logique $^{(75)}$. Eberhard considère toutefois que l'État doit formellement conserver un droit de regard sur l'enseignement de la religion. Sa réflexion est motivée notamment par des considérations historiques:

" Les évolutions consécutives au bouleversement de 1918 ont conduit à la séparation de l'école et de l'Église (cf. art. 144, 146, 149 de la Constitution de Weimar). La voix de la conscience, conscience de l'État et conscience de la civilisation, s'entend toutefois dans l'intégration de l'enseignement religieux au nombre des disciplines obligatoires à l'école (art. 149): par là, l'État a évité la rupture radicale avec l'histoire scolaire allemande, et il prémunit le patrimoine de la société religieuse effective contre tout arbitraire doctrinal, de quelque côté qu'il vienne » ${ }^{(76)}$.

70 R. Scherwatzky, « Hegel in der religiösen Krisis der Gegenwart » (note 66), p. 268.

71 Ibid.: «Ce n'est pas sans raison que des esprits aussi importants que Frick, Spranger, Natorp et d'autres identifient le fond de la crise actuelle dans le domaine de la religion (et non de la politique!). Ce faisant, ils se situent au niveau de pensée que s'était choisi Hegel. »

72 K. Kesseler, « Kulturproblematik und Religionsproblematik » (note 54), p. 240.

73 Chez Spranger und Kerschensteiner par exemple, la doctrine hégélienne de l'Esprit objectif aurait supplanté « la philosophie de Kant, qui jusque-là dominait tout »: voir Heinrich MAtThes, "Das Problem des Erlebnisunterrichts », MERU, 24 (1931), p. 153-174, sp. 155-156.

74 Ibid., p. 157.

75 Otto Eberhard, " Kirche und Schule in ihrer Lebensverbundenheit », MERU, 22 (1929), p. 204-206, sp. 205.

76 Ibid. 
Du reste, l'école confessionnelle constitue une école " séculière » au sens de la Constitution de Weimar, dès lors qu'on la considère du point de vue de la doctrine des deux règnes: sa mission est en effet celle d'un service public rendu au peuple ${ }^{(77)}$.

Le second compromis de Weimar n'avait quant à lui été qu'effleuré par Eberhard. Busch, un pasteur de Dresde, mène quant à lui l'analyse approfondie du $\$ 149$ de la Constitution. L'Assemblée constituante n'a selon lui pas eu l'intention de lier les enseignants à des principes strictement confessionnels. Au contraire, la Constitution de Weimar se caractérise par son sens de la diversité des confessions: les principes (Grundsätze) de l'enseignement ne sont pour elle que des "énoncés servant de fondements » (Grund-Sätze). L'Église protestante, continue Busch, n'est pas liée à une approche particulière de la Bible, non plus qu'à des confessions de foi ou à une ecclésiologie déterminée; elle ne reconnaît aucune "théologie normale", au sens de normative ${ }^{(78)}$. Busch attend donc à son tour de l'État qu'il préserve l'enseignement religieux des empiètements des Églises.

La question posée par la croissance du nombre d'écoles confessionnelles sur le territoire allemand semble elle-même s'être clarifiée à la fin de la République de Weimar. Le pasteur saxon August Umland explique ainsi les progrès du «cléricalisme » par une intensification selon lui malsaine de la conscience confessionnelle. La fondation d'associations et autres sociétés protestantes constitue un « recul » quand on se rappelle que le monde associatif catholique avait autrefois été regardé avec distance ${ }^{(79)}$. Umland rend hommage à l'approche ecclésiologique d'Otto Piper, qui tente d'introduire un concept méta-confessionnel de l'autorité de l'Église sans pour autant sacrifier l'ancrage confessionnel de sa réflexion. La démarche de Piper permet selon Umland de rejeter toute idée de magistère extérieur: le centre de gravité de l'action ecclésiale ne se trouve pas dans le monde associatif confessionnel, mais dans la théologie ${ }^{(80)}$. Dans cette perspective, un rôle particulier revient à un enseignement (académique) soutenu par l'État ${ }^{(81)}$.

La discussion ecclésiologique ne s'est pas arrêtée à la fin des années vingt. Les réflexions de Hermann Schuster le montrent qui, contre la tentative de Holstein pour construire un lien "pneumatique » entre l'Église et l'école, se réclame de Sohm, pour qui « l'idée d'un droit ecclésiastique protestant est fondamentalement incompatible avec l'essence de l'Église protestante pensée comme communauté de foi " ${ }^{(82)}$.

On a vu plus haut le rôle qu'une pensée de l'État a pu elle aussi tenir dans la réflexion sur l'école et l'Église. Elle a ouvert l'espace dans lequel une approche réflexive et responsable des formes futures de la relation entre enseignement religieux et Église a pu se déployer, dans l'abandon conscient de toute forme normative de théologie en protestantisme.

77 Ibid.

78 Dr. Busch, « Die Grundsätze der evangelischen Religionsgesellschaft », MERU, 23 (1930), p. 30-34, sp. 32 .

79 August Umland, « Neuere Versuche zur Überwindung des Kirchenproblems », MERU, 22 (1929), p. 193-202, sp. 193-194.

80 Ibid., p. 195: « Pour Piper l’Église est la science de Dieu sur la Terre. »

81 Ibid., p. 193-194.

82 Hermann Schuster, «Zur religiösen Lage der Gegenwart », ZevRU, 1930, p. 135-140, sp. 135-136. 


\section{Conclusion}

Et finalement? Que pouvons-nous retirer de cette analyse critique de la situation sous Weimar? Le travail historique a pour fonction de faire renaître dans la mémoire les liens du passé et du présent. De ce point de vue, le résultat peut-être le plus important est en même temps le moins visible: l'enseignement de la religion n'a pu trouver sa place dans la Constitution de Weimar que parce que celle-ci garantissait parallèlement l'instruction civique et la «formation au travail » - conçue, dans ses débuts, comme une discipline autonome. Par là, la Constitution de Weimar donnait à l'enseignement de la religion un pendant qui, sous cette forme au moins, a disparu de la Loi fondamentale de 1949. Les suites d'un tel constat sont encore difficilement évaluables: ce serait le sujet d'une autre étude.

La question du rôle joué par les pensées de l'État dans les discussions de la République de Weimar sur l'éducation religieuse apparaît ainsi dans toute sa complexité. Deux modèles émergent, le premier issu de Kant (l'État fournit le cadre dans lequel peut s'exercer le droit de l'individu à une formation religieuse), le second de Hegel (l'État défend la liberté religieuse contre les empiètements cléricaux). Ces deux modèles ont peu perdu de leur actualité. Si la liberté (religieuse) de l'individu reste au centre des préoccupations, les difficultés que pose sa transposition scolaire reçoivent plusieurs solutions dans la Loi fondamentale de la RFA: s'agit-il pour l'État de défendre l'enseignement religieux selon des préceptes kantiens, pour protéger la liberté religieuse de ses citoyens $(\$ 4 \mathrm{GG})^{(83)}$, ou sa mission est-elle, en termes plus hégéliens, de garantir le maintien des spécificités d'une religion donnée en raison du potentiel historique et culturel qu'elle détient ${ }^{(84)}$ ? Dans leurs diverses variantes, ces deux modèles restent présents dans les discussions menées en RFA, y compris après 1989.

Traduit de l'allemand par Philippe Büttgen

\section{Zusammenfassung}

Der Beitrag beschäftigt sich mit der Rolle, die dem Nachdenken über den Staat in den religionspädagogischen Diskursen der Weimarer Republik zukam und arbeitet dabei die unterschiedlichen rechtsphilosophischen Einflüsse - die Tradition Hegels einerseits, die Tradition Kants andererseits - heraus. Der Analyse der theoretischen Konzeptionen von Staat folgt die Erörterung entsprechender Auswirkungen auf das Nachdenken über Religionsunterricht: die verschiedenen Diskurse strebten zunehmend eine "Aussetzung" der durch die Weimarer Verfassung vollzogenen Trennung von Kirche und Staat an.

83 Bernhard Dressler renvoie à l'art. 4 de la Loi fondamentale pour justifier ce qu'il considère comme l'exercice actif d'un droit démocratique fondamental: voir Unterscheidungen. Religion und Bildung, Leipzig, EVA, 2006, p. 180-181.

84 Rainer Lachmann voit dans l'art. 149 de la Constitution de Weimar repris dans l'art. 7, 3 de la Loi fondamentale une formule de compromis particulièrement heureuse : voir " Die Weimarer Republik » (note 7), p. 206, ainsi que B. Dressler, Unterscheidungen (note 83), p. 181-182. 